\title{
Microbiology Susceptibility Specimen Type
}

National Cancer Institute

\section{Source}

National Cancer Institute. Microbiology Susceptibility Specimen Type. NCI Thesaurus.

Code C162351.

The type of material sample used for microbiology susceptibility testing. 